\title{
ARTICLE
}

Received 10 Jun 2013 | Accepted 6 Dec 2013 | Published 15 Jan $2014 \quad$ DOI: 10.1038/ncomms4069

\section{WDM-compatible mode-division multiplexing on a silicon chip}

\author{
Lian-Wee Luo ${ }^{1, \star}$, Noam Ophir ${ }^{2, \star}$, Christine P. Chen ${ }^{2}$, Lucas H. Gabrielli ${ }^{1}$, Carl B. Poitras ${ }^{1}$, \\ Keren Bergmen ${ }^{2} \&$ Michal Lipson ${ }^{1,3}$
}

Significant effort in optical-fibre research has been put in recent years into realizing mode-division multiplexing (MDM) in conjunction with wavelength-division multiplexing (WDM) to enable further scaling of the communication bandwidth per fibre. In contrast, almost all integrated photonics operate exclusively in the single-mode regime. MDM is rarely considered for integrated photonics because of the difficulty in coupling selectively to high-order modes, which usually results in high inter-modal crosstalk. Here we show the first microring-based demonstration of on-chip WDM-compatible mode-division multiplexing with low modal crosstalk and loss. Our approach can potentially increase the aggregate data rate by many times for on-chip ultrahigh bandwidth communications.

\footnotetext{
${ }^{1}$ School of Electrical and Computer Engineering, Cornell University, 428 Phillips Hall, Ithaca, New York 14853, USA. ${ }^{2}$ Department of Electrical Engineering, Columbia University, 500 West 120th Street, New York, New York 10027, USA. ${ }^{3}$ Kavli Institute at Cornell for Nanoscale Science, Cornell University, 420 Physical Sciences Building, Ithaca, New York 14853, USA. * These authors contributed equally to this work. Correspondence and requests for materials should be addressed to M.L. (email: ML292@cornell.edu).
} 
C urrent-integrated photonics operate almost exclusively in the single-mode regime and utilize wavelength-division multiplexing (WDM $)^{1,2}$, which supports limited scalability in bandwidth density. In contrast, fibre communications are increasingly targeting multimode operation in conjunction with WDM to further scale the communication bandwidth transmitted per fibre ${ }^{3}$. Multimode communications in fibres have been demonstrated with space-division multiplexing in multi-core fibres $^{4-8}$ or mode-division multiplexing (MDM) in few-mode fibres $^{9-17}$ and have exploited each spatial mode as an independent channel. It is similarly of interest to attempt to incorporate the spatial-mode-parallelism degree of freedom into complex photonic-integrated circuits to introduce additional functionality and provide potential performance gains. In particular, one application of interest for photonic-integrated circuits is on-chip and off-chip high-bandwidth-density interconnects. Here we present a platform enabling MDM in conjunction with WDM for such on-chip photonic networks. This platform could increase the bandwidth density of on-chip interconnects without increasing the number of waveguides, waveguide crossings (which can dominate the loss in complex interconnects ${ }^{18,19}$ ) and chip area, as well as add an additional design degree of freedom in future photonic-integrated circuits and networks.

Some of the key challenges of realizing on-chip MDM-enabled interconnects lie in creating mode (de)multiplexers with low modal crosstalk and loss, which also support WDM (a key feature of many integrated-optics interconnect designs). Previous implementations of on-chip mode multiplexers based on Mach-Zehnder interferometers ${ }^{20,21}$, Multimode Interference couplers $^{22-24}$, asymmetric directional couplers ${ }^{25-27}$ and y-junctions ${ }^{28-30}$ typically had large footprints, complex and strict design limitations or only supported a limited number of optical modes. A compact and reconfigurable mode (de)multiplexer that can be straight-forwardly scaled to support numerous modes is essential for realizing MDM-WDM in integrated photonics.

In the following sections, we detail the first microringbased demonstration of an on-chip WDM-compatible modedivision multiplexing (and demultiplexing) scheme. The system performance of this scheme with all of its associated stages (multiplexing, transmission and demultiplexing) is characterized with multiple co-propagating $10 \mathrm{~Gb} \mathrm{~s}^{-1}$ high-speed communication signals reaching up to $60 \mathrm{Gbs}^{-1}$ of aggregate bandwidth.

\section{Results}

Selective mode coupling by phase matching. We demonstrate on-chip MDM-WDM by engineering the propagation constants of high-confinement photonic structures in order to enable selective coupling to different spatial modes at different wavelengths. The silicon photonic platform is attractive for implementing this approach as the propagation constants of the different spatial modes can be engineered to differ significantlythanks to the high core-cladding $\left(\mathrm{Si} / \mathrm{SiO}_{2}\right)$ index contrast. We choose a waveguide height for which the confinement is high, and therefore widely different propagation constants can be achieved by varying the waveguide width. Figure la shows that for a given 250-nm-tall silicon waveguide a large range of effective indices from 2.0 to 2.9 can be achieved corresponding to the propagation constants of the $\mathrm{TE}_{0}$ (transverse electric) through $\mathrm{TE}_{4}$ spatial modes at $\lambda=1,550 \mathrm{~nm}$. On the basis of propagation constant matching, an optical mode in a single-mode waveguide can be evanescently coupled to a specific spatial mode in an adjacent multimode waveguide, in which the coupling strength to the mode depends on the width of the multimode waveguide.

In order to realize a compact and tunable MDM device, we employ single-mode microring resonators to selectively couple to the different spatial modes in a multimode waveguide. To simultaneously support multiple WDM channels in the MDM scheme, we design the free-spectral range of the microrings to match the wavelength channel spacing. The mode multiplexer and demultiplexer, which are designed to operate in the quasi-TE mode, comprise of three identical microrings coupled to a multimode waveguide (see Fig. 1b). Each microring, formed from a 450-nm-wide waveguide, is designed to support only the fundamental TE mode with an effective index of 2.46. The multimode transport waveguide comprises several sections with tapering widths ranging from $450 \mathrm{~nm}$ to $1.41 \mu \mathrm{m}$. When the multimode waveguide width corresponds to $450 \mathrm{~nm}, 930 \mathrm{~nm}$ or $1.41 \mu \mathrm{m}$, the effective indices of $\mathrm{TE}_{0}, \mathrm{TE}_{1}$ or $\mathrm{TE}_{2}$ modes, respectively, match the effective index of the $\mathrm{TE}_{0}$ mode of the microrings $\left(n_{\text {eff }}=2.46\right)$ and therefore couple efficiently to the microrings. The three insets in Fig. 1b show such coupling of the $\mathrm{TE}_{0}$ mode of the microring to the $\mathrm{TE}_{0}, \mathrm{TE}_{1}$ or $\mathrm{TE}_{2}$ modes in the multimode waveguide.

In order to achieve good device performance, the coupling through the microrings has to minimize the insertion loss for the desired mode as well as minimize the power coupled to the other modes. This can be achieved by optimizing the coupling gap and coupler length between the microrings and the waveguides. a

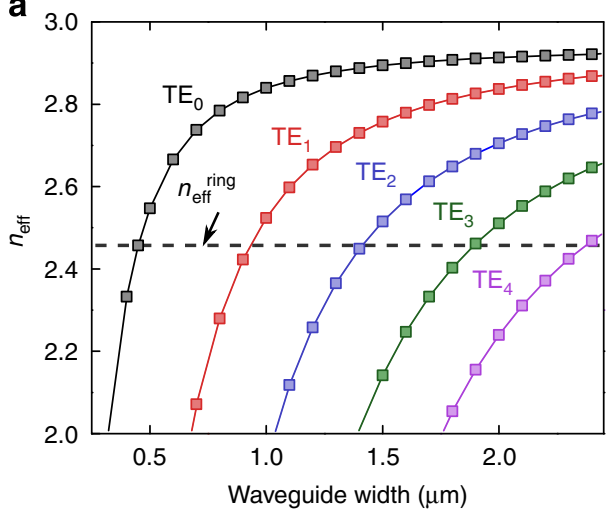

b

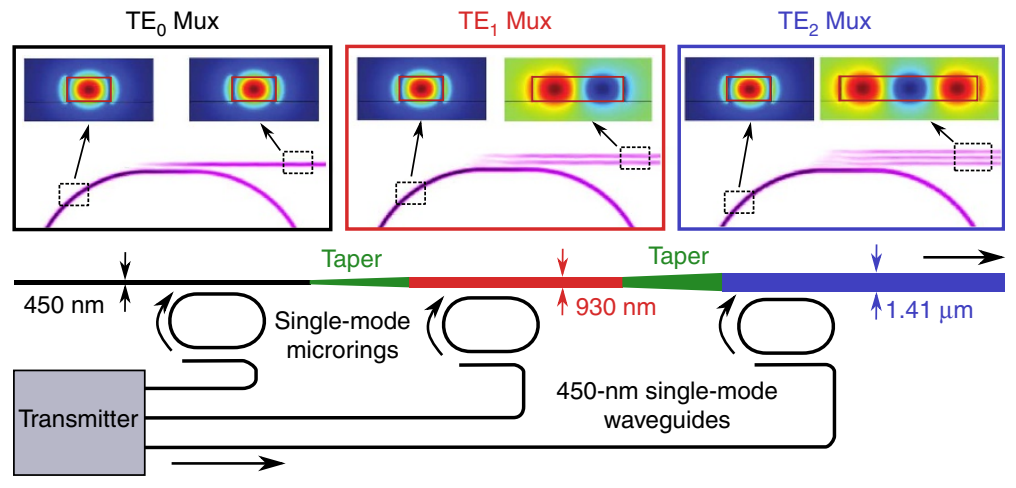

Figure 1 | Phase-matching condition for 250-nm-tall silicon waveguides. (a) Simulated effective index of the optical modes in waveguide of different widths at $\lambda=1,550 \mathrm{~nm}$. (b) Selective coupling of the single-mode microrings to a specific spatial mode in the multimode waveguide, with each section of the multimode waveguide linked by adiabatic tapered waveguides. The insets show the selective coupling of each multiplexer ( $T E_{0}$, $T E_{1}$ and $T E_{2}$ ). 
a

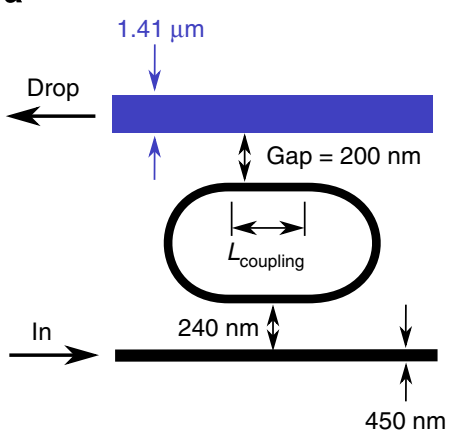

b

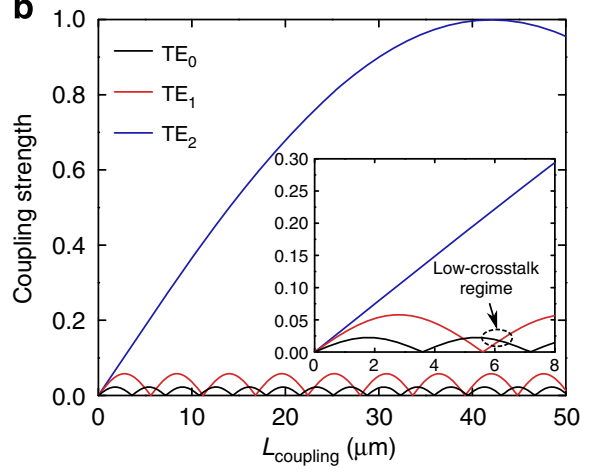

C

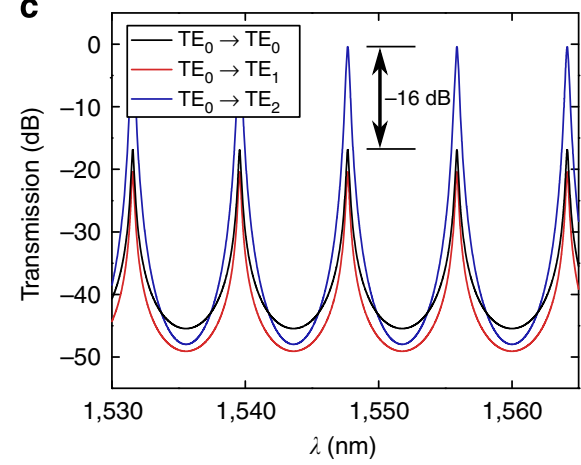

Figure 2 | Coupling strength of the three spatial TE modes of a 1.41- $\mu \mathrm{m}$-wide waveguide to the $\mathrm{TE}_{\mathrm{o}}$ mode of a 450-nm-wide microring waveguide. (a) Schematic of an add-drop microring with asymmetric input and drop waveguides. (b) Calculated coupling strength of the coupling region between the microring and the multimode waveguides. The inset shows the zoom-in coupling strength for coupling length $<8 \mu \mathrm{m}$. A short coupling length (low coupling strength) is sufficient to achieve critical coupling between the single-mode input waveguide and the multimode drop waveguide via the microring. (c) Simulated transmission and crosstalk levels at the drop port with an optimized coupling length of $6 \mu \mathrm{m}$.

For example, with a 200-nm gap (see Fig. 2a), the power coupled from the microring to the modes of a $1.41-\mu \mathrm{m}$ waveguide can be computed based on the coupled-mode analysis (see Methods) as depicted in Fig. 2b. With the microring-based design, a short coupling length is feasible as only a relatively low coupling strength is required to transfer all the power from the singlemode input waveguide to the $\mathrm{TE}_{2}$ mode in the multimode drop waveguide via the microring (typically termed critical coupling $)^{31}$. The coupling to the undesired modes $\left(\mathrm{TE}_{1}\right.$ and $\left.\mathrm{TE}_{0}\right)$ is much weaker because of the phase mismatch of these modes, and thus a $6-\mu \mathrm{m}$-long coupling length can achieve optimal loss and crosstalk performance (see Fig. 2c).

We design the microring resonance linewidth to be at least $15-\mathrm{GHz}$ in order to enable $10-\mathrm{Gb} \mathrm{s}^{-1}$ data transmission with negligible signal degradation. We also include an integrated heater on top of each microring to tune the microring resonances to align to the WDM channels and thereby optimize the performance of the device ${ }^{32}$. This design can be easily modified to handle additional phase-matched modes by widening the multimode waveguide (Fig. 1a). In this particular design scheme, the multimode transport waveguide width is varied, requiring the lowest order mode to be added first and dropped last. However, one could in principle keep the multimode waveguide width constant and instead vary the microring waveguide width, enabling the addition and dropping of arbitrary modes without disturbing neighbouring modes. Another direct extension of this design is the inclusion of additional sets of identical microrings coupled to each multimode waveguide section to enable wavelength-channel grooming directly on each spatial mode.

Optical performance of the fabricated device. The fabrication details for this MDM device (Fig. 3a) are documented in the Methods section. We image the optical modes at the output of the multimode waveguide to verify the excitation of the different spatial modes (see Methods for the imaging experimental details). We observe well-defined $\mathrm{TE}_{0}, \mathrm{TE}_{1}$ and $\mathrm{TE}_{2}$ modes as predicted by simulation (Fig. 3b). From the spectral transmission scans for each combination of input and output ports, we can quantify the amount of crosstalk resulting from the spatial mode multiplexing and demultiplexing. Figure $3 \mathrm{c}$ shows the transmission spectrum at output port 1 (see port definitions in Fig. 3a) from each input. The measured insertion loss of this port at $\lambda=1,547 \mathrm{~nm}$ is $13 \mathrm{~dB}$ and the optical crosstalk (defined as the ratio of desired signal power to the sum of the interfering channels' power) is $-22 \mathrm{~dB}$. The insertion loss of port 2 at $\lambda=1,547 \mathrm{~nm}$ is $19 \mathrm{~dB}$ and the optical crosstalk is $-18 \mathrm{~dB}$ (Fig. $3 \mathrm{~d}$ ). The insertion loss of port 3 at $\lambda=1,547 \mathrm{~nm}$ is $26 \mathrm{~dB}$ and crosstalk is $-12 \mathrm{~dB}$ (Fig. 3e). The main contribution to the insertion loss is the aggregate $10-\mathrm{dB}$ fibre-to-chip coupling loss. The rest of the insertion loss is attributed to the waveguide propagation loss and microring intrinsic loss. The higher insertion loss in port 3 compared with the other two ports is because of a suboptimal microring coupling gap. By ensuring critical coupling between the waveguides and microrings, achievable on-chip losses of this device are expected to total around $1.5 \mathrm{~dB}$.

As detailed in the previous section, the crosstalk can be minimized by optimizing the coupling length between the microring and the multimode waveguide to reduce coupling of the undesired modes (see Fig. 2b). We expect the crosstalk at the output port 3 of the current device to be less than $-16 \mathrm{~dB}$ for an optimized coupling length of $6 \mu \mathrm{m}$ (the fabricated coupler was only $5 \mu \mathrm{m}$ long) (see Fig. $2 \mathrm{c}$ ). The crosstalk can be further reduced by introducing weaker coupling at the microring-multimode waveguide coupling region (by having a larger coupling gap of $280 \mathrm{~nm}$ ) to lower the maximum coupling of the undesired modes. This in turn results in a longer coupling length $\left(L_{\text {coupling }} \approx 11 \mu \mathrm{m}\right)$ to maintain the critical coupling condition and from our calculations should enable devices with less than $-30 \mathrm{~dB}$ of crosstalk.

High-speed MDM link with three spatial modes. We simultaneously launch a single $10-\mathrm{Gb} / \mathrm{s}$ data channel into all the three input ports of the mode multiplexer and measure a small power penalty $\left(<1.9 \mathrm{~dB}\right.$ measured at a BER of $\left.10^{-9}\right)$ on each output port of the mode demultiplexer. The experimental setup for performance evaluation is illustrated in Fig. 4a. In order to measure these power penalties, the laser channel at $1,563 \mathrm{~nm}$ is modulated with PRBS $2^{31}-1$ on-off-keyed data by an amplitude modulator and then further phase-imprinted with a sweptfrequency sinusoid in order to enable bit-error-rate (BER) measurements on channels that experience coherent crosstalk (further information is provided in the Methods section). The data signal is then amplified, split evenly between the three input ports of the on-chip mode multiplexer and simultaneously injected in quasiTE polarization to the multiplexer ports. The varying fibre spans leading to the device ensure that the data are decorrelated between the ports (see Methods). The demultiplexed signals are recovered one at a time for inspection on a DCA and BER evaluation. Error-free transmission (BER $<10^{-12}$ ) and open eye diagrams (Fig. 4b,c) are observed for all the signals at the three 
a

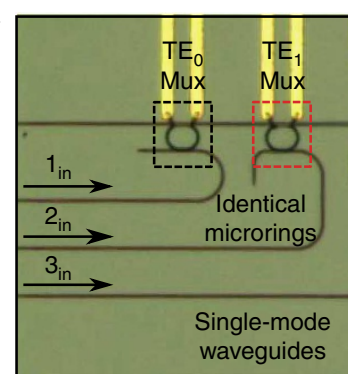

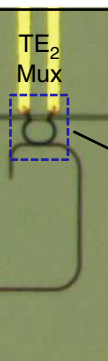

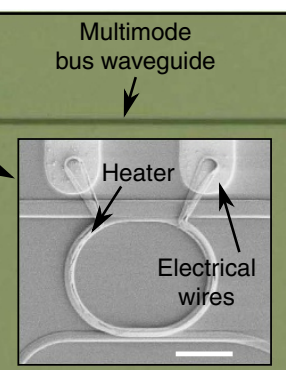

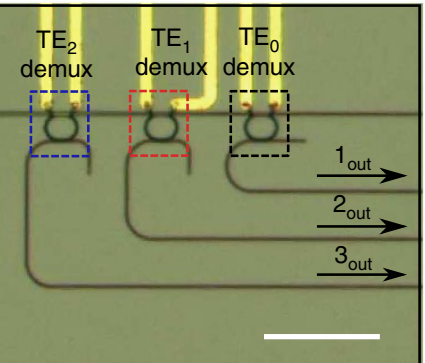

b

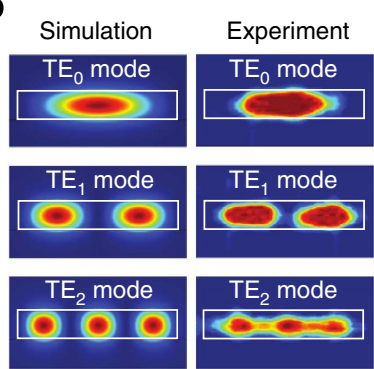

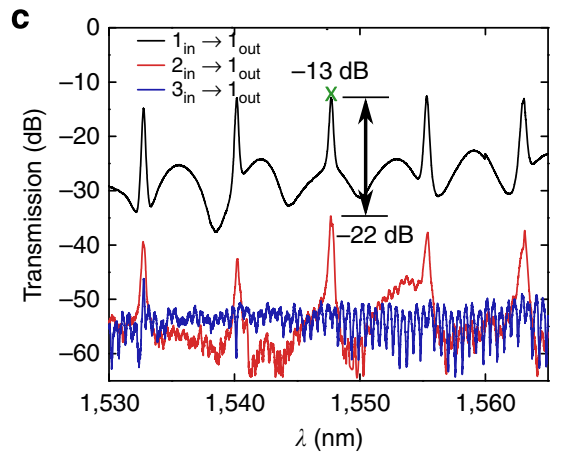
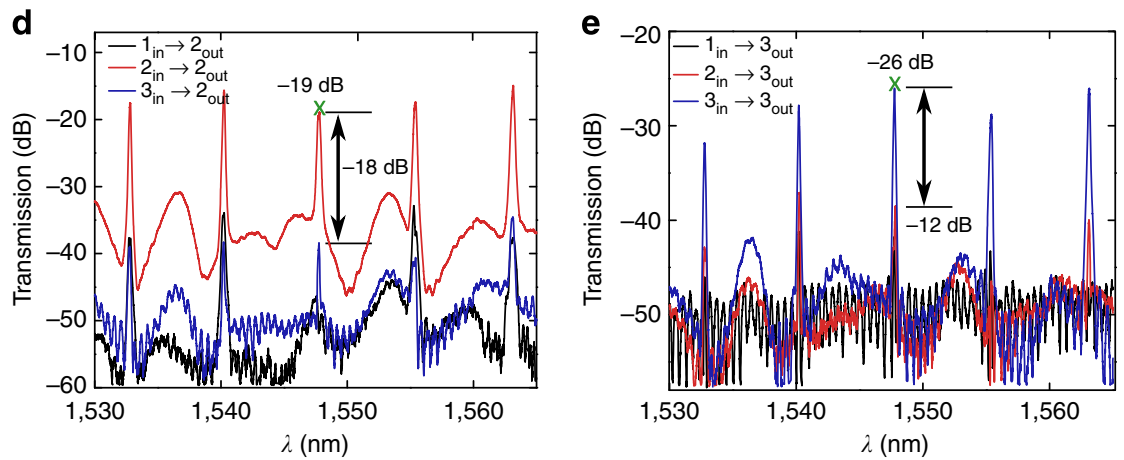

Figure 3 | Optical performance of the fabricated device. (a) Microscope image of the fabricated device (100 $\mu \mathrm{m}$ scale). Inset: scanning electron microscope (SEM) image showing the heater to tune each individual microring resonator (10 $\mu \mathrm{m}$ scale). (b) Simulated and experimental images of the optical modes at the cross-section of the multimode waveguide. (c-e) Optical transmission and crosstalk at the three output ports for signal injection on each of the three input ports.
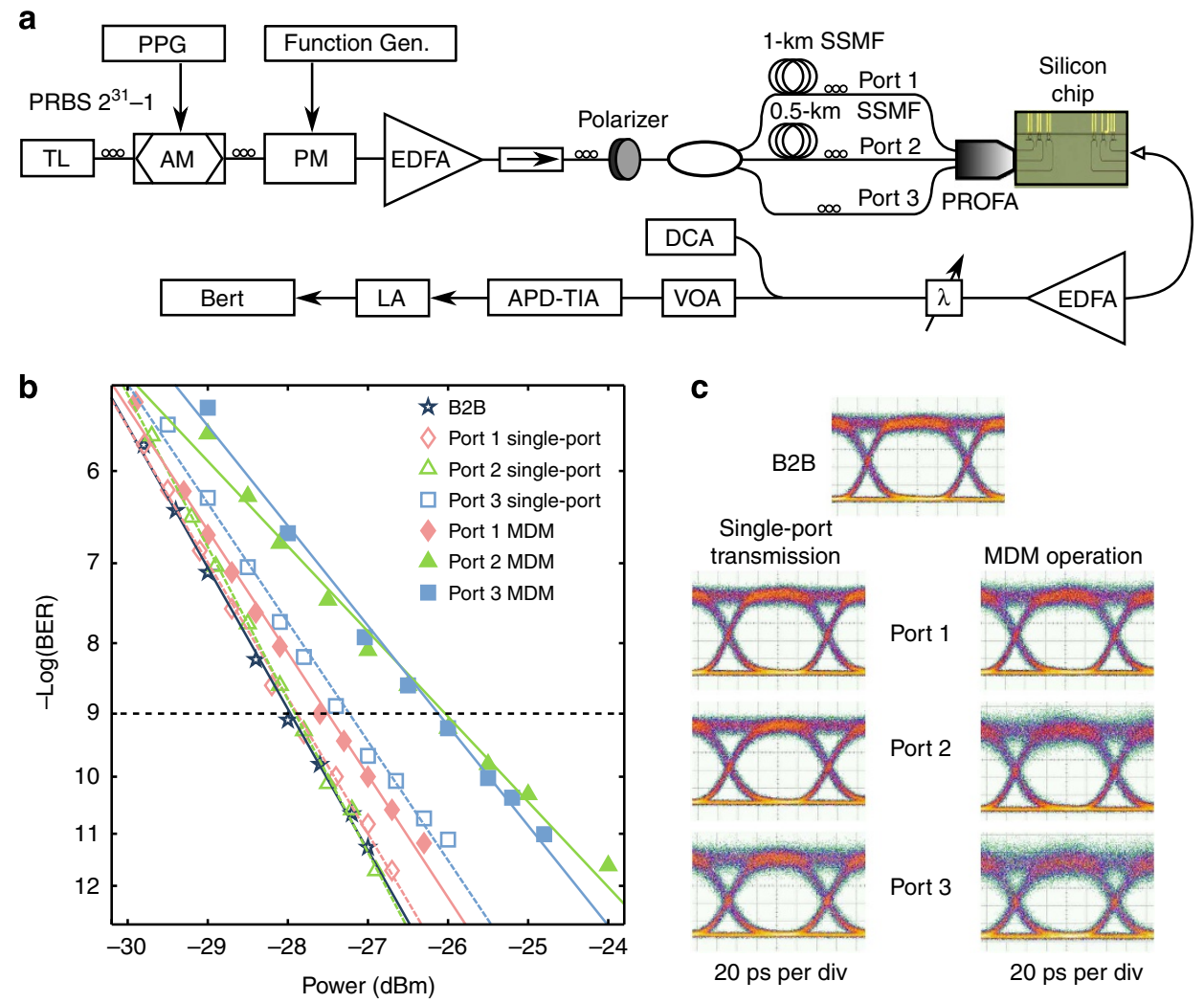

Figure 4 | 3-Mode $\times \mathbf{1 0} \mathbf{~ G b ~ s}^{-1}$ multiplexing link. (a) Experimental setup for performance evaluation including Pulsed Pattern Generator (PPG), Tunable Laser (TL), Amplitude Modulator (AM), Phase Modulator (PM), Erbium-Doped Fibre Amplifier (EDFA), Isolator $(\rightarrow)$, Standard Single-Mode Fibre (SSMF), Tunable Filter ( $\lambda$ ), Digital-Communications Analyzer (DCA), Variable Optical Attenuator (VOA), Avalanche-Photodiode (APD-TIA), Limiting Amplifier (LA) and Bit-Error-Rate Tester (BERT). (b) BER measurements for back-to-back (B2B) test case, single port transmission and MDM operation for all three ports. (c) Corresponding eye diagrams for the inspected signals. 
output ports. To account for fabrication imperfections, we improve the performance of port 3 at the expense of increased crosstalk and spectral filtering penalties on port 2 by wavelength detuning the $\mathrm{TE}_{1}$ multiplexer microring. This enables device operation with an overall balanced power penalty of $1.9 \mathrm{~dB}$ on ports 2 and 3 and $0.5 \mathrm{~dB}$ on port 1 . In order to verify that the intrachannel crosstalk is indeed the main mechanism of signal degradation $^{33}$, we also inspect the channel performance with only one input port injected at a time. We observe that the single-input transmission results in $0.1 \mathrm{~dB}$ penalties on ports 1 and 2 and $0.8 \mathrm{~dB}$ on port 3 (with the higher penalty on port 3 resulting from the higher insertion loss through this port, which leads to a larger OSNR degradation at the post-chip EDFA). Therefore, we conclude that crosstalk is the main contributing factor to signal degradation in this device. The penalties are measured relative to a back-to-back reference case, which is defined and measured by replacing the chip with a tunable attenuator set to replicate the fibre-to-fibre loss of the lowest insertion-loss port.

MDM-WDM link with two spatial modes and three WDM channels. We measure a low $(<1.4 \mathrm{~dB})$ power penalty for joint MDM-WDM operation by launching three different $10-\mathrm{Gb} / \mathrm{s}$ wavelength channels spanning the full C-band into two input ports of the multiplexer (ports 1 and 2). A modified setup (depicted in Fig. 5a) is used to correctly decorrelate the wavelength channels (see Methods), and polarizers are included in the experiment to ensure that all the wavelength channels are launched on chip at the quasi-TE polarization with equal power. We set the wavelength channels to span the full C-band (limited by the EDFA gain band) and the microrings are tuned onresonance to maximize power transmission at $1,547 \mathrm{~nm}$. The power penalties for both ports vary between 0.6 and $1.4 \mathrm{~dB}$ for all the three wavelength channels (Fig. 5b) with performance variation attributed to slightly varying levels of crosstalk for the different wavelength channels. Error-free transmission (BER $<10^{-12}$ ) and open eye diagrams (Fig. 5c) are observed for all the three channels at the two output ports. These results show that only a minimal penalty is added by extending the device operation to support WDM concurrently with the MDM.

\section{Discussion}

In conclusion, we have shown a platform that enables on-chip MDM-WDM optical interconnection for ultrahigh bandwidth communications. Our simulation in Fig. 1a showed that when the multimode waveguide width tapers up to $2.37 \mu \mathrm{m}$, five spatial modes can be supported by this platform. In principle using a wider waveguide one could support an even larger number of modes. Each microring resonator in practice is able to support 87 WDM channels over the entire C-band (1,530-1,565 nm) by increasing the microring size such that the channel spacing is $50 \mathrm{GHz}$. Therefore, the on-chip MDM-WDM platform with the above-mentioned dimension design can potentially support an aggregate data rate up to $4.35 \mathrm{~Tb} \mathrm{~s}^{-1}$ with five spatial modes and 87 WDM channels.

\section{Methods}

Device design and fabrication. We fabricate the reconfigurable MDM-WDM silicon microring resonators on a $250 \mathrm{~nm}$ SOI wafer with $3 \mu \mathrm{m}$ of buried oxide using standard CMOS fabrication processes. We patterned the waveguides using e-beam lithography with the dimensions as followed: the input/output ports and the microring are $450 \mathrm{~nm}$ wide; the $\mathrm{TE}_{0}, \mathrm{TE}_{1}$ and $\mathrm{TE}_{2}$ (de)multiplexers multimode transport waveguide are $450 \mathrm{~nm}, 930 \mathrm{~nm}$ and $1.41 \mu \mathrm{m}$ wide, respectively, and each (de)multiplexer is linked by an adiabatic taper of $80 \mu \mathrm{m}$ long. All the microrings have a radius of $10 \mu \mathrm{m}$ and a coupling length of $5 \mu \mathrm{m}$. The separation gap between the microrings and all the input waveguides is $240 \mathrm{~nm}$, while the separation gaps between the microrings and $\mathrm{TE}_{0}, \mathrm{TE}_{1}$ and $\mathrm{TE}_{2}$ (de)multiplexer multimode waveguide are $240 \mathrm{~nm}, 200 \mathrm{~nm}$ and $200 \mathrm{~nm}$, respectively. The silicon waveguides are then etched, followed by the e-beam resist being stripped and the etched structures are clad with a $1-\mu \mathrm{m}$-thick silicon oxide layer using plasma-enhanced chemical vapour deposition to confine the optical mode. $\mathrm{NiCr}(300 \mathrm{~nm})$ is subsequently

a

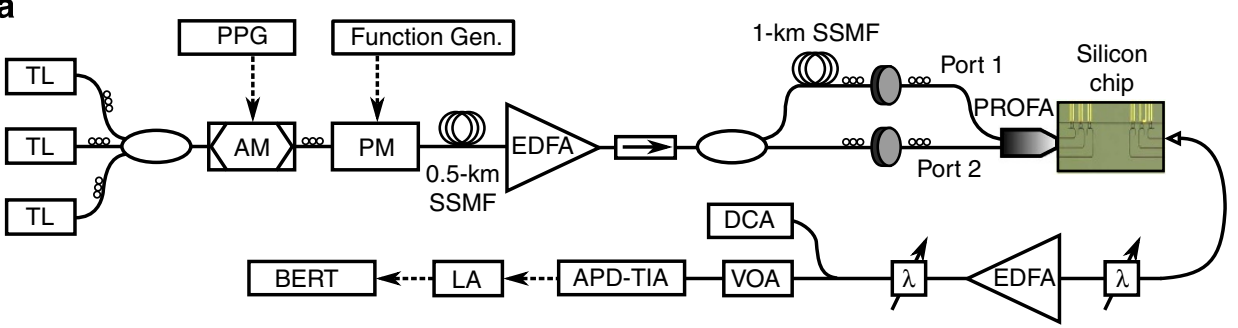

b

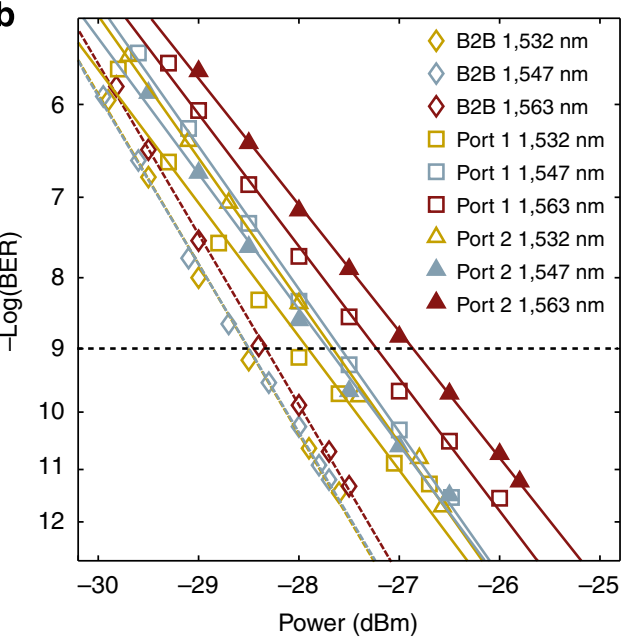

C
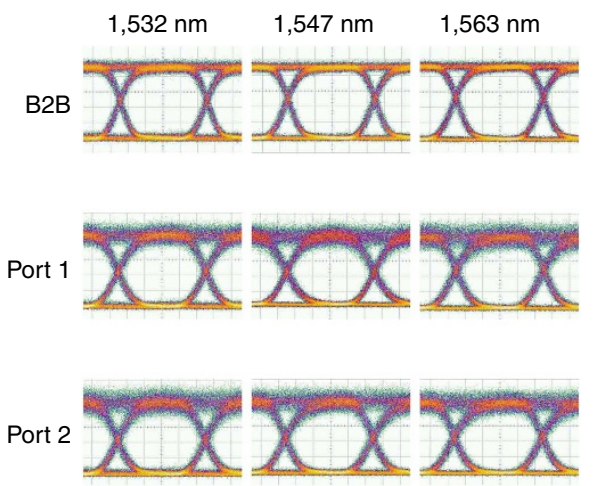

20 ps per div

Figure 5 | 2-Mode $\times$ 3-wavelength $\times \mathbf{1 0} \mathbf{G b ~ s}^{-1}$ MDM-WDM link. (a) Experimental setup for demonstrating combined MDM and WDM operation. (b) BER measurements for B2B test cases and full MDM-WDM operation for both ports. (c) Corresponding eye diagrams for the inspected signals. 
evaporated on the microring resonators above the cladding to create the $1-\mu \mathrm{m}$ wide heaters. Finally, $500 \mathrm{~nm}$ of gold $(\mathrm{Au})$ are evaporated to define the electrical wires and contact pads using a lift-off process. The final fabricated device is illustrated in Fig. 3a. The footprint of this device is $0.11 \mathrm{~mm}^{2}$ excluding the electrical wires.

Imaging of the spatial modes in the multimode waveguide. We fabricate a test device that only has the mode multiplexer section $\left(\mathrm{TE}_{0}, \mathrm{TE}_{1}\right.$ and $\left.\mathrm{TE}_{2}\right)$ and is terminated with the $1.41-\mu \mathrm{m}$ wide multimode waveguide. We couple a $1,547-\mathrm{nm}$ laser (on-resonance of each microring) into one of the input ports at one time. The output spatial modes of the multimode waveguide are then magnified with a $\times 40$ aspheric lens and imaged on an infrared camera as shown in Fig. $3 \mathrm{~b}$.

Coupled-mode analysis for asymmetric coupled waveguides. Coupled-mode theory for two weakly coupled optical modes relates the complex amplitudes of the modes $a_{1}$ and $a_{2}$ through a set of differential equations ${ }^{34}$ :

$$
\begin{aligned}
& \frac{\mathrm{d} a_{1}}{\mathrm{~d} z}=-j \beta_{1} a_{1}+\kappa_{12} a_{2}, \\
& \frac{\mathrm{d} a_{2}}{\mathrm{~d} z}=-j \beta_{2} a_{2}+\kappa_{21} a_{1},
\end{aligned}
$$

the solutions to this equation set, assuming the waves $a_{1}(0)$ and $a_{2}(0)$ are launched at $z=0$, are given by:

$$
\begin{aligned}
& a_{1}(z)=\left[a_{1}(0)\left(\cos \beta_{0} z+j \frac{\beta_{2}-\beta_{1}}{2 \beta_{0}} \sin \beta_{0} z\right)+\frac{\kappa_{12}}{\beta_{0}} a_{2}(0) \sin \beta_{0} z\right] e^{-j\left[\left(\beta_{1}+\beta_{2}\right) / 2\right] z}, \\
& a_{2}(z)=\left[\frac{\kappa_{21}}{\beta_{0}} a_{1}(0) \sin \beta_{0} z+a_{2}(0)\left(\cos \beta_{0} z+j \frac{\beta_{1}-\beta_{2}}{2 \beta_{0}} \sin \beta_{0} z\right)\right] e^{-j\left[\left(\beta_{1}+\beta_{2}\right) / 2\right] z},
\end{aligned}
$$

where

$$
\beta_{0}=\sqrt{\left(\frac{\beta_{1}-\beta_{2}}{2}\right)^{2}+\kappa_{12} \kappa_{21}} .
$$

If the initial waves $a_{1}(0)=1$
to $a_{2}$ is given by $\left|\frac{\kappa_{21}}{\beta_{0}} \sin \beta_{0} z\right|$.

Phase dithering. Intrachannel crosstalk ${ }^{33}$ results in coherent interference of the laser with itself. In a test setup not employing any phase decoherence mechanisms, this results in a slow change of the output signal power as the phases leading to the device under test change as a result of thermal fluctuations in the fibres. If this remains untreated, these power fluctuations (on the temporal order of multiple seconds) prevent accurate BER measurements over short time spans. In order to enable finite-time BER measurements, two mechanisms are employed simultaneously to average out the slow phase fluctuations: (1) the arms leading to the multiplexer input ports are decorrelated by at least $0.5 \mathrm{~km}$ Standard SingleMode Fibre. This is close to the $1-\mathrm{km}$ coherence length of the $200-\mathrm{kHz}$ linewidth lasers we used in the experiments, therefore ensuring some phase decoherence of the signals. (2) In order to guarantee full-phase orthogonality regardless of the intrinsic laser linewidth, we incorporate phase modulation of a repeating linearly chirped signal consisting of a frequency sweep from 20 to $10 \mathrm{MHz}$ over a $5-\mathrm{ms}$ period. With a $0.5-\mathrm{km}$ path difference (roughly 2.5 -s relative delay), the phase difference between adjacent ports oscillates over $2 \pi$ at $5 \mathrm{kHz}$, guaranteeing averaging of the phase difference in power measurements averaged over $100 \mathrm{~ms}$.

Multiport edge coupling. Employing a method similar to one previously reported ${ }^{35}$ we couple to three input ports simultaneously using a Pitch Reducing Optical Fibre Array (PROFA) mounted on a fully angle-adjustable stage. The PROFA alignment was optimized to be within $2 \mathrm{~dB}$ of the optimal coupling values for all the ports simultaneously. Output coupling is performed with a tapered lensed fibre aligned to one output port at a time.

Intrachannel crosstalk penalties. The back-to-back reference test case for power penalty measurements is defined by bypassing the chip and emulating insertion loss for the lowest loss port (port 1) with a variable optical attenuator. Intrachannel crosstalk penalties result in a power penalty predicted analytically ${ }^{33}$ as $\mathrm{PP}=-10 \log _{10}(1-2 \sqrt{\varepsilon})$ where $\varepsilon$ is the ratio of the desired-signal's power to interfering signals' powers.

Channel decorrelation. In order to guarantee correct characterization of the device, the input data channels that originate from a single Pulsed Pattern Generator need to be decorrelated. The data channels coupled to the ports of the device are decorrelated by 0.5 -and $1-\mathrm{km}$-long fibre delays, which ensure that the patterns are relatively shifted between ports by at least $24 \mathrm{~kb}$ out of the pattern length of $2 \mathrm{~Gb}\left(2^{31}-1\right.$ PRBS). In the second experiment, the wavelength channels are first decorrelated using the dispersion of a $0.5-\mathrm{km}$ fibre to achieve at least 90-bit relative delay between adjacent wavelength channels. The inputs to the two ports used in this experiment are also decorrelated by a fixed 1-km fibre delay, which guarantees decorrelation between the ports used.

\section{References}

1. Nagarajan, R. et al. Large-scale photonic integrated circuits. J. Sel. Top. Quant. Electron. 11, 50-65 (2005).

2. Jalali, B. \& Fathpour, S. Silicon photonics. J. Lightw. Technol. 24, 4600-4615 (2006).

3. Richardson, D. J., Fini, J. M. \& Nelson., L. E. Space-division multiplexing in optical fibres. Nat. Photonics 7, 354-362 (2013).

4. Sakaguchi, J. et al. in Optical Fibre Communication Conference and Exposition (OFC/NFOEC), 2011 and the National Fibre Optic Engineers Conference, paper PDPB6 (Los Angeles, CA, USA, 2011).

5. Feuer, M. D. et al. in Optical Fibre Communication Conference, paper PDP5B.8 (Anaheim, CA, USA, 2013).

6. Sakaguchi, J. et al. in Optical Fibre Communication Conference, paper PDP5C.1 (Los Angeles, CA, USA, 2012).

7. Takara, H. et al. in European Conference and Exhibition on Optical Communication, paper Th.3.C.1 (Amsterdam, Netherlands, 2012).

8. Qian, D. et al. in Frontiers in Optics, paper FW6C.3 (Rochester, NY, USA, 2012).

9. Randel, S. et al. $6 \times 56-\mathrm{Gb} / \mathrm{s}$ mode-division multiplexed transmission over $33-\mathrm{km}$ few-mode fibre enabled by $6 \times 6$ MIMO equalization. Opt. Express. 19, 16697-16707 (2011).

10. Hanzawa, N. et al. in Optical Fibre Communication Conference and Exposition (OFC/NFOEC), 2011 and the National Fibre Optic Engineers Conference, paper OWA4 (Los Angeles, CA, USA, 2011).

11. Ryf, R. et al. Mode-division multiplexing over $96 \mathrm{~km}$ of few-mode fibre using coherent $6 \times 6$ MIMO processing. J. Lightw. Technol. 30, 521-531 (2012).

12. Salsi, M. et al. Mode-division multiplexing of $2 \times 100 \mathrm{~Gb} / \mathrm{s}$ channels using an LCOS-based spatial modulator. J. Lightw. Technol. 30, 618-623 (2012).

13. Amin, A. A. et al. Dual-LP ${ }_{11}$ mode $4 \times 4$ MIMO-OFDM transmission over a two-mode fibre. Opt. Express. 19, 16672-16679 (2011).

14. Bai, Neng. et al. Mode-division multiplexed transmission with inline few-mode fibre amplifier. Opt. Express. 20, 2668-2680 (2012).

15. Ryf, R. et al. in Optical Fibre Communication Conference, paper PDP5A.1 (Anaheim, CA, USA, 2013).

16. Ip, E. et al. in National Fibre Optic Engineers Conference, paper PDP5A.2 (Anaheim, CA, USA, 2013).

17. Li, A., Amin, A. A., Chen, X. \& Shieh, W. Transmission of 107-Gb/s mode and polarization multiplexed CO-OFDM signal over a two-mode fibre. Opt. Express. 19, 8808-8814 (2011).

18. Chan, J., Hendry, G., Biberman, A. \& Bergman, K. Architectural exploration of chip-scale photonic interconnection network designs using physical-layer analysis. J. Lightw. Technol. 28, 1305-1315 (2010).

19. Chan, J. \& Bergman, K. Photonic interconnection network architectures using wavelength-selective spatial routing for chip-scale communications. J Opt. Commun. Network 4, 189-201 (2012).

20. Bagheri, S. \& Green, W. in 6th IEEE International Conference on Group IV Photonics, 166-168 (2009).

21. Huang, Y., Xu, G. \& Ho, S.-T. An ultracompact optical mode order converter. Phot. Technol. Lett. 18, 2281-2283 (2006).

22. Kawaguchi, Y. \& Tsutsumi, K. Mode multiplexing and demultiplexing devices using multimode interference couplers. Electron Lett. 38, 1701-1702 (2002).

23. Uematsu, T., Ishizaka, Y., Kawaguchi, Y., Saitoh, K. \& Koshiba, M. Design of a compact two-mode multi/demultiplexer consisting of multimode interference waveguides and a wavelength-insensitive phase shifter for mode-division multiplexing transmission. J. Lightw. Technol. 30, 2421-2426 (2012).

24. Leuthold, J., Hess, R., Eckner, J., Besse, P. A. \& Melchior, H. Spatial mode filters realized with multimode interference couplers. Opt. Lett. 21, 836-838 (1996).

25. Greenberg, M. \& Orenstein, M. Multimode add-drop multiplexing by adiabatic linearly tapered coupling. Opt. Express. 13, 9381-9387 (2005).

26. Ding, Y. et al. On-chip two-mode division multiplexing using tapered directional coupler-based mode multiplexer and demultiplexer. Opt. Express. 21, 10376-10382 (2013).

27. Dai, D., Wang, J. \& Shi, Y. Silicon mode (de) multiplexer enabling high capacity photonic networks-on-chip with a single-wavelength-carrier light. Opt. Lett. 38, 1422-1424 (2013).

28. Lee, B.-T. \& Shin, S.-Y. Mode-order converter in a multimode waveguide Opt. Lett. 28, 1660-1662 (2003).

29. Love, J. D. \& Riesen, N. Single-, few-, and multimode y-junctions. J. Lightw Technol 30, 304-309 (2012).

30. Driscoll, J. B. et al. Asymmetric y junctions in silicon waveguides for on-chip mode-division multiplexing. Opt. Lett. 38, 1854-1856 (2013). 
31. Yariv, A. Universal relations for coupling of optical power between microresonators and dielectric waveguides. Electron Lett. 36, 321-322 (2000).

32. Sherwood-Droz, N. et al. Optical $4 \times 4$ hitless slicon router for optical networks-on-chip (NOC). Opt. Express 16, 15915-15922 (2008).

33. Ramaswami, R., Sivarajan, K. \& Sasaki, G. Optical Networks: a Practical Perspective (Morgan Kaufmann, 2009).

34. Haus, H. A. Waves and Fields in Optoelectronics, Vol. 1, Prentice-Hall (1984).

35. Doany, F. E. et al. Multichannel high-bandwidth coupling of ultradense silicon photonic waveguide array to standard-pitch fiber array. J. Lightw. Technol. 29, 475-482 (2011).

\section{Acknowledgements}

We gratefully acknowledge support from the US Air Force (AFOSR) program FA955009-1-0704 on 'Robust and Complex on-chip Nanophotonics' supervised by Dr Gernot Pomrenke, support from DARPA for award no. W911NF-11-1-0435 supervised by Dr Jagdeep Shah. This material is based upon work supported by the National Science Foundation under Grant No. 1143893. This work was supported in part by the NSF through CIAN ERC under Grant EEC-0812072 and was performed in part at the Cornell NanoScale Facility, a member of the National Nanotechnology Infrastructure Network, which is supported by the NSF. We also acknowledge the support from the NSF and Semiconductor Research Corporation under grant ECCS-0903406 SRC Task 2001.

Lian-Wee Luo acknowledges a fellowship from the Agency of Science, Technology and Research $\left(A^{\star}\right.$ STAR), Singapore.

\section{Author contributions}

L.-W.L. and N.O. contributed equally to this work. L.-W.L. designed and fabricated the device with the assistance of L.H.G. in the simulations. L.-W.L., N.O., C.P.C. and C.B.P. conducted the experiments together. L.-W.L., N.O., K.B. and M.L. discussed the results and implications. L.-W.L., N.O., K.B. and M.L. contributed to the writing of this paper.

\section{Additional information}

Competing financial interests: The authors declare no competing financial interests.

Reprints and permission information is available online at http://npg.nature.com/ reprintsandpermissions/

How to cite this article: Luo, L.-W. et al. WDM-compatible mode-division multiplexing on a silicon chip. Nat. Commun. 5:3069 doi: 10.1038/ncomms4069 (2014). 